\title{
Disparate Cellular Basis of Improved Liver Repair in $\beta$-Catenin-Overexpressing Mice After Long-Term Exposure to 3,5-Diethoxycarbonyl-1,4-Dihydrocollidine
}

\author{
Michael D. Thompson, ${ }^{*}$ Prince Awuah, ${ }^{*}$ \\ Sucha Singh, ${ }^{*}$ and Satdarshan P.S. Monga*† \\ From the Departments of Pathology, ${ }^{*}$ and Medicine, ${ }^{\dagger}$ University \\ of Pittsburgh, School of Medicine, Pittsburgh, Pennsylvania
}

\begin{abstract}
Administration of a hepatotoxic diet containing $0.1 \%$ 3,5-diethoxycarbonyl-1,4-dihydrocollidine (DDC) induces biliary damage followed by hepatocyte injury, which is repaired through atypical ductular proliferation and oval cells and their subsequent differentiation to bile duct cells and hepatocytes. In this study, we examine whether excess $\beta$-catenin in transgenic (TG) mice would provide any reparative advantage in response to DDC. No differences in appearance or numbers of total A6-positive oval cells were observed after DDC administration. However, an increase in A6-positive "atypical hepatocytes" in the TG livers was observed after 14 and 28 days, coinciding with an increase in proliferating cell nuclear antigen-positive hepatocytes. Intriguingly, after chronic DDC administration for $\mathbf{1 5 0}$ days, a further increase in atypical hepatocytes was evident in TG mice, with higher numbers of proliferating cell nuclear antigen-positive hepatocytes exhibiting cytoplasmic/nuclear $\beta$-catenin and $\alpha$-fetoprotein but not CK19, HNF1及, or Trop-2. Coincidently, we observed an improvement in intrahepatic cholestasis as seen by decreases in both serum bilirubin and alkaline phosphatase levels in TG mice, indicating an overall improvement in hepatic repair. TG mice exposed to DDC for 4 weeks followed by 2 days of normal chow showed decreases in alkaline phosphatase, atypical ductular proliferation, and periportal inflammation compared with wild-type animals, verifying improved biliary repair in TG livers. Thus, we report a potential role of $\beta$-catenin in liver repair, especially in enhancing the resolution of intrahepatic cholestasis after DDC injury. (Am J Pathol 2010, 177:1812-1822; DOI: 10.2353/ajpath.2010.100173)
\end{abstract}

Despite being first identified more than 50 years ago, adult liver progenitor cells (oval cells) remain an enigma.
Little is known about the molecular mechanisms that drive their activation and proliferation. Although such cells are scarce in healthy liver, they can be found adjacent to the terminal ducts of the biliary tree. ${ }^{1}$ These cells are generally quiescent and only arise in situations in which the innate proliferative capacity of the hepatocytes and cholangiocytes is impaired or overwhelmed. Indeed, oval cell activation is observed in conditions associated with chronic liver injury and development of hepatocellular carcinoma such as chronic viral hepatitis, alcoholic liver disease, and nonalcoholic fatty liver disease., ${ }^{2,3}$ Greater than $50 \%$ of human hepatocellular carcinomas express one or more markers of progenitor cells or oncofetal proteins such as $\alpha$-fetoprotein and cytokeratins 7 , 14 , or $19 .{ }^{4-6}$ This observation is even more relevant because approximately half of the small cell dysplastic foci, the earliest cancer precursor lesions known to date, consist of progenitor cells and their progeny, suggesting that these lesions are the result of activation and proliferation of progenitor cells. ${ }^{7}$ Another report has shown that half of hepatocellular adenomas consist of progenitor cells and intermediate hepatocytes. ${ }^{8}$ Given the association with liver cancer, great interest lies in molecular characterization of this cell population to potentially target them for therapy.

One pathway that plays a role in the oval cell response is the $\mathrm{Wnt} / \beta$-catenin pathway. $\beta$-Catenin translocates to the nucleus and activates transcription of various target genes after binding of Wht ligand to frizzled at the cell membrane and a series of phosphorylation events that lead to the dissociation of $\beta$-catenin from the Axin/Apc/ GSK3 $\beta$ degradation complex. Previous studies have shown a conclusive role for $\beta$-catenin in hepatocyte maturation and differentiation during hepatic development. ${ }^{9-11}$ We have

\footnotetext{
Supported by the National Institutes of Health (grants 1R01-DK62277 and 1R01-CA124414 to S.P.S.M. and 1F30-DK083235 to M.D.T.) and by Rango's Fund for the Enhancement of Pathology Research.

Accepted for publication June 16, 2010.

Address reprint requests to Satdarshan P. S. Monga, M.D., Director, Division of Experimental Pathology, Associate Professor of Pathology and Medicine, University of Pittsburgh School of Medicine, 200 Lothrop St., S-421 BST, Pittsburgh, PA 15216. E-mail: smonga@pitt.edu.
} 
reported that immature hepatocytes or progenitors possess greater nuclear and cytoplasmic localization of $\beta$-catenin in comparison with more differentiated hepatocytes, in which $\beta$-catenin is mostly located at the hepatocyte membrane in association with E-cadherin and C-Met, which was also evident during Matrigel-induced hepatocyte differentiation in primary cultures. ${ }^{12}$ We and others demonstrated the direct importance of Wnt/ $\beta$-catenin signaling in hepatic oval cell biology in rat and mouse models. ${ }^{13,14}$

Various models are currently used to study the characteristics of oval cells in vivo, including the 3,5-diethoxycarbonyl-1,4-dihydrocollidine (DDC) diet model used in mice. ${ }^{15,16}$ Chronic DDC exposure leads to significant hepatic injury, with ongoing repair prompted by atypical ductular proliferation and oval cells or hepatic progenitors. ${ }^{16,17}$ In the current study, we compare and contrast the response of control or $\beta$-catenin transgenic mice to short-term (4 weeks) and long-term (150 days) DDC exposure. Interestingly, we found that expression of the $\beta$-catenin transgene in hepatocytes led to improved resolution of intrahepatic cholestasis that was coincident with an increase in A6-positive hepatocytes and a greater number of patent bile ducts after long-term DDC exposure. This finding suggests an overall greater ability of the transgenic livers to resolve the DDC-induced hepatic injury especially by enhanced biliary repair.

\section{Materials and Methods}

\section{Animals}

We have previously generated and characterized a $\beta$-catenin transgenic mouse on the FVB background that expresses the human $\beta$-catenin gene under the albumin promoter/enhancer, which carries a mutation affecting serine residue $45 .{ }^{18}$ Only male mice were used for all experiments and age-matched wild-type (WT) FVB mice served as controls for all experiments. At the time of sacrifice, a portion of the excised liver was fixed in 10\% neutral buffered formalin and processed for paraffin embedding. A portion of liver was frozen in Tissue-Tek OTC compound for frozen sections. The remaining liver was quickly frozen in liquid nitrogen and stored at $-80^{\circ} \mathrm{C}$. All animal studies were performed in strict accordance with the guidelines of the Institutional Animal Use and Care Committee at the University of Pittsburgh School of Medicine and the National Institutes of Health.

\section{DDC Diet Feeding}

Mice were fed a special diet containing 0.1\% DDC (Bioserve, Frenchtown, $\mathrm{NJ}$ ) for periods of time ranging from 3 to 150 days to induce atypical ductular proliferation, which has been described previously. ${ }^{17}$ For the recovery study, mice were fed the DDC diet for 28 days followed by reinstitution of normal chow for 2 days, at which time animals were sacrificed.

\section{Serum Biochemistry}

Serum biochemical measurements were performed by the University of Pittsburgh Department of Pathology Laboratory Support Services. Total bilirubin, alkaline phosphatase, aspartate aminotransferase (AST), and alanine aminotransferase (ALT) were measured in samples taken before sacrifice at multiple time points.

\section{Immunohistochemistry}

Tissues fixed in 10\% formalin were embedded in paraffin, and $4-\mu \mathrm{m}$ sections cut onto Superfrost Plus glass slides (Fisher Scientific, Pittsburgh, PA) were used for H\&E or immunohistochemical analysis as described elsewhere. ${ }^{9}$ Primary antibodies used include proliferating cell nuclear antigen (PCNA) (1:4000, Santa Cruz Biotechnology), $\beta$-catenin (1:50, Santa Cruz Biotechnology), CD45 (1: 100, Santa Cruz Biotechnology), $\alpha$-fetoprotein (1:50, Santa Cruz Biotechnology), Trop-2 (1:7, R\&D Systems, Minneapolis, MN), cytokeratin (CK) $19(1 ; 100$, Santa Cruz Biotechnology), and hepatic nuclear factor (HNF) $1 \beta$ (1: 50, Santa Cruz Biotechnology). For quantification of PCNA- and CD45-positive cells, 5 high-power field images were taken around a periportal region for each animal and counted using Zeiss AxioVision software. All counts were averaged among each group (transgenic [TG] or WT) at each time point.

\section{Immunofluorescence}

For analysis of $\mathrm{A} 6$ and $\mathrm{CEBP} \alpha$ expression, frozen sections were fixed with acetone for 10 minutes, washed in PBS, and incubated with $2 \%$ normal donkey serum prepared in $1 \%$ bovine serum albumin in PBS for 20 minutes to suppress nonspecific IgG binding. Rat anti-A6 (1:100; a kind gift from Dr. Valentina Factor, National Cancer Institute, Bethesda, MD) or rabbit anti-CEBP $\alpha$ (1:50, Santa Cruz Biotechnology) was prepared in $1 \%$ bovine serum albumin in PBS and applied to sections for 1 hour at room temperature. After being washed with PBS, sections were incubated in the dark with Cy2-conjugated donkey anti-rat secondary antibody (1:500) in 1\% bovine serum albumin in PBS for 1 hour. Then after another wash with PBS, sections were counterstained and mounted using mounting medium containing 4',6-diamidino-2phenylindole. Fluorescent microscopy was performed on a Zeiss Axioscope microscope. For quantification of A6positive cells, 5 high-power field images were taken around a periportal region for each animal and counted using Zeiss Axiovision software. All counts were averaged among each group (TG or WT) at each time point.

\section{Statistical Analysis}

All experiments were performed with three or more animals, and representative data are presented. Quantification of positive cells (A6, PCNA, and CD45) and serum biochemistry measurements were compared for statistical analysis by Student's $t$-test (Microsoft Excel), and $P<$ 
A

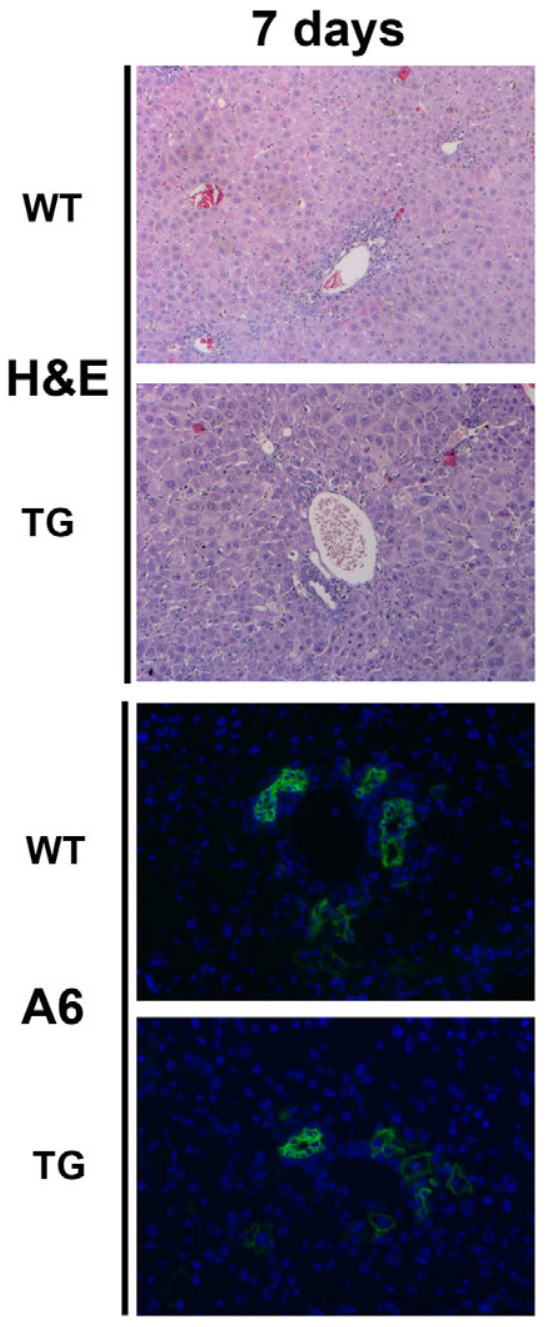

B

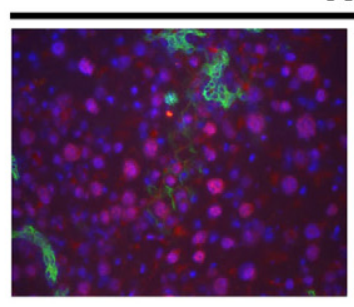

WT
14 days
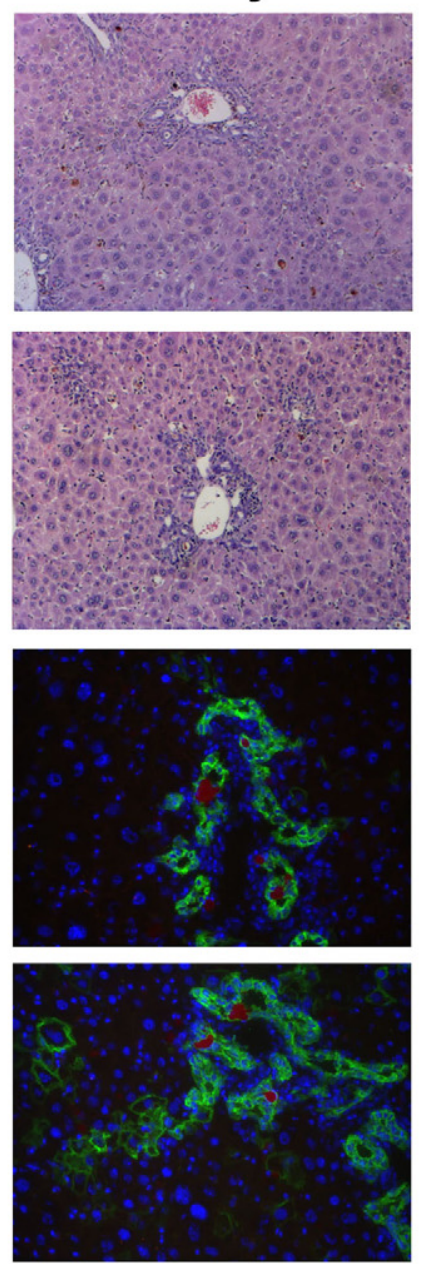

28 days
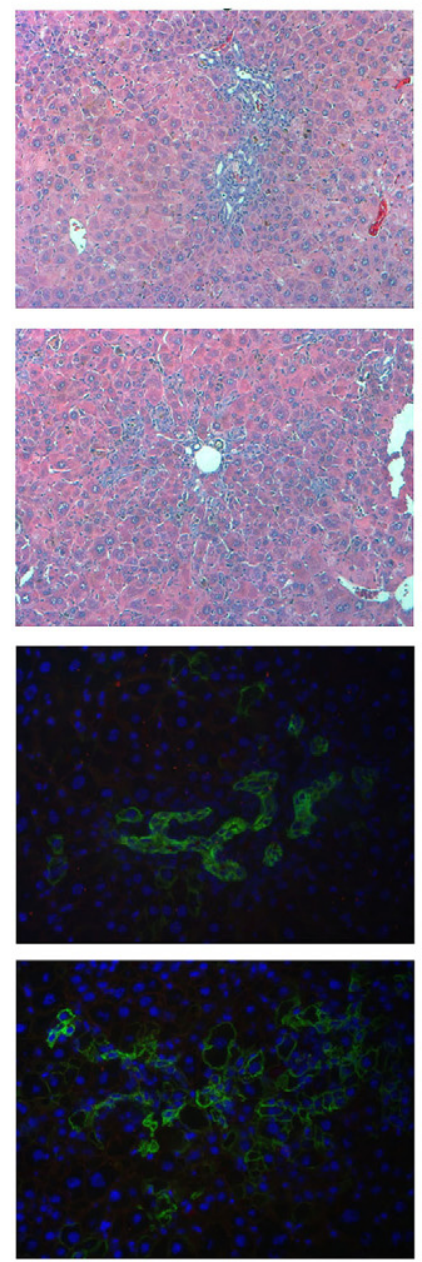

TG

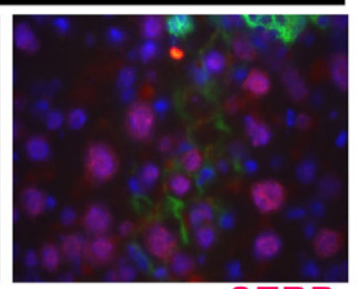

\section{CEBP $\alpha$ and $\mathrm{A} 6$}

C

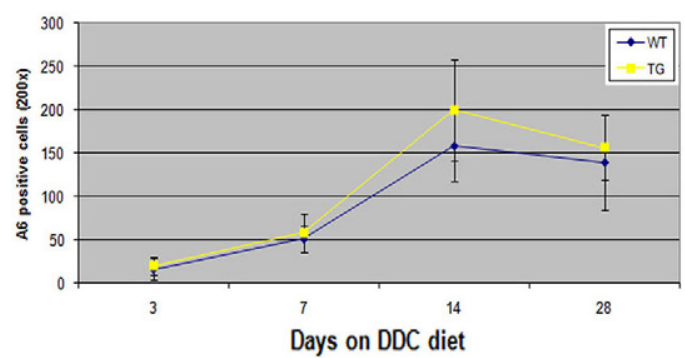

D

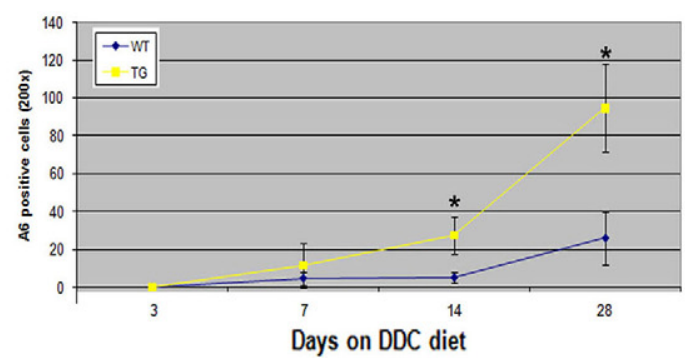

Figure 1. No changes occur in ductular responses to DDC with overexpression of $\beta$-catenin, but morphological disparity among the A6-positive population is present. A: Representative photomicrographs of H\&E and A6 immunofluorescence staining (green) of livers from WT and TG mice after 7, 14 , and 28 days of feeding with the DDC diet. Original magnification: $\times 100$ (H\&E); $\times 200$ (A6). 4',6-Diamidino-2-phenylindole (blue) was used to stain nuclei in immunofluorescence images. B: Atypical hepatocytes, mostly evident in TG livers at 28 days, coexpress A6 (green) and CEBP $\alpha$ (red) by immunofluorescence (original magnification $\times 200$ (left panels); $\times 400$ (right panels). C: Quantification of total A6-positive cells in WT and TG livers after 3, 7, 14, and 28 days of DDC feeding. No significant difference was observed at any of the time points between WT and TG livers. D: Quantification of A6-positive hepatocytes in WT and TG livers after 3, 7, 14, and 28 days of DDC feeding. More A6-positive hepatocytes were observed in TG than in WT livers at 14 and 28 days. ${ }^{*} P<0.01$. 
0.05 or 0.01 was considered significant or extremely significant, respectively.

\section{Results}

Cellular Disparity Among the A6-Positive

Population Despite Comparable ADP in WT and TG Mice after Short-Term DDC Exposure

TG and WT mice on the FVB background were fed the DDC diet for time periods ranging from 3 to 28 days to examine the ADP response in the presence of excess $\beta$-catenin. No differences in histology and serum biochemistry measurements for bilirubin, AST, and ALT were observed between the two groups (Figure $1 \mathrm{~A}$ and data not shown). Initial analysis of the response to DDC was performed by staining for $A 6$, a ductular and progenitor marker. Surprisingly, we observed no change in the number of A6-positive cells between WT and TG mice between 3 and 28 days (Figure 1, A and C). We did, however note a disparity in the morphology of A6-positive cells between the two groups at 14 and 28 days (Figure $1 \mathrm{~A})$. Whereas the majority of A6-positive cells in WT mice
A

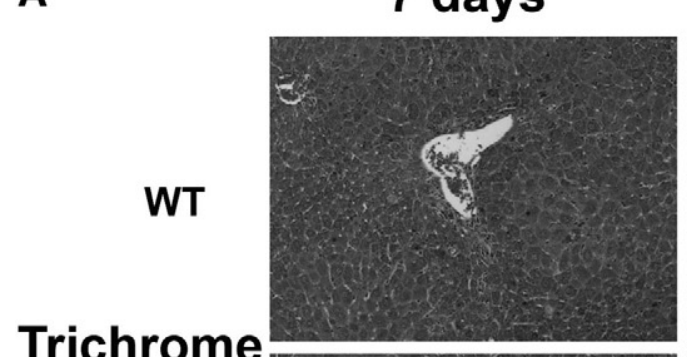

Trichrome

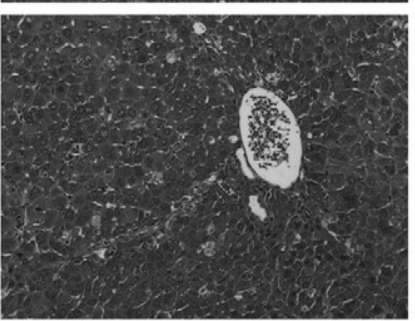

TG

PCNA

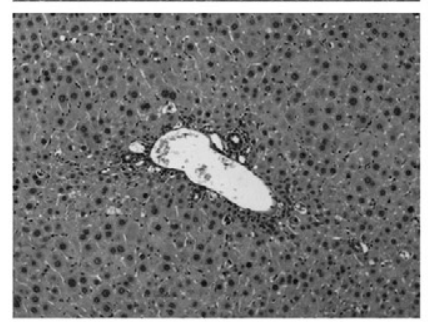

TG

B
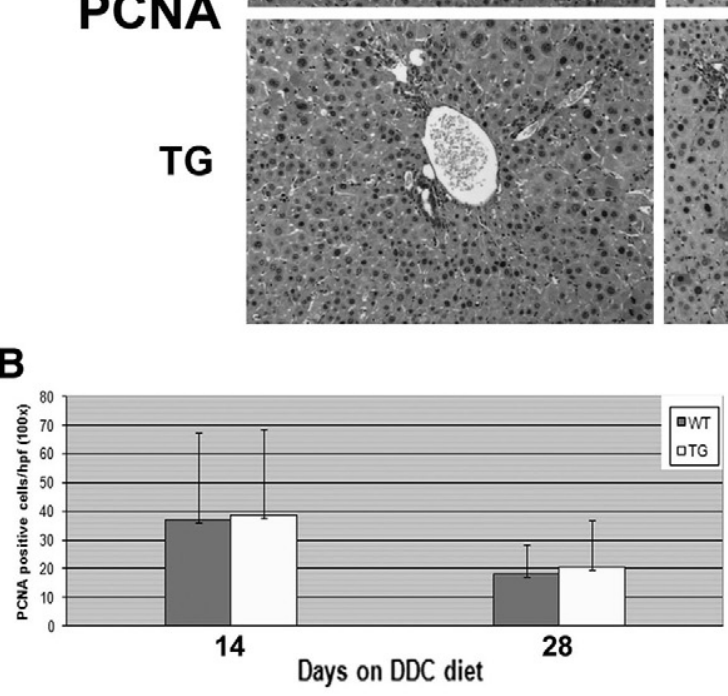

14 days
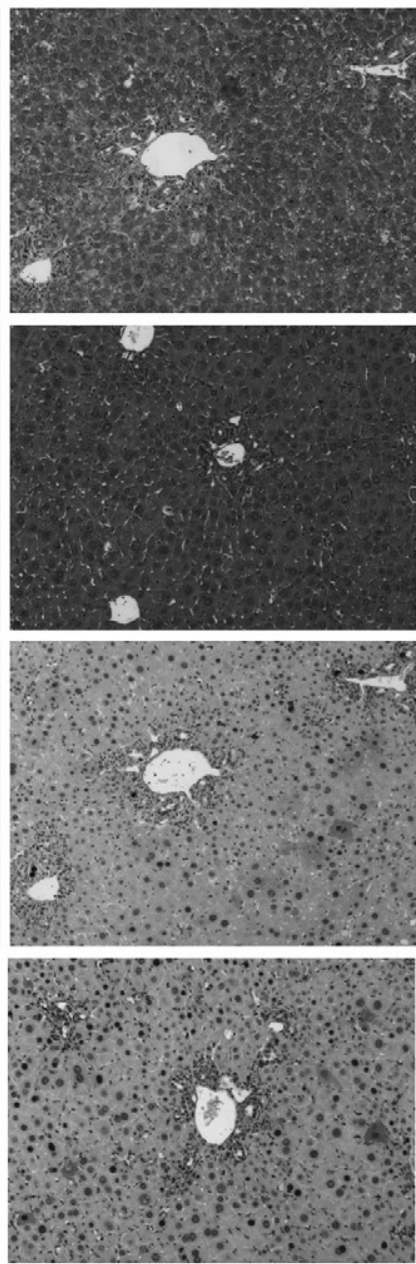

C

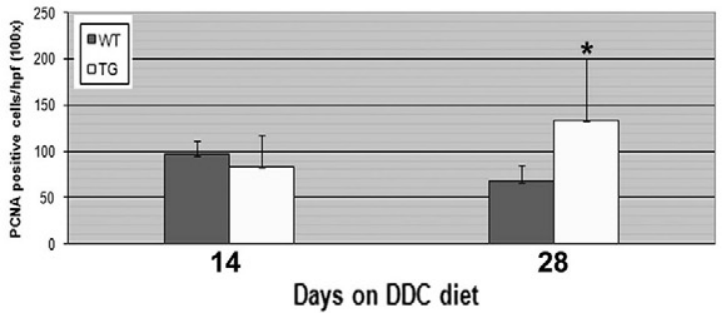

Figure 2. An increase in hepatocyte proliferation, but no change in fibrosis was observed in TG livers after short-term DDC exposure. A: Representative photomicrographs for trichrome (fibrosis, blue) and PCNA staining (proliferation, brown) of livers from WT and TG mice after 7, 14 , and 28 days of feeding with the DDC diet. Original magnification, $\times 100$. B: Quantification of PCNA-positive biliary epithelial cells at 14 and 28 days of DDC feeding shows no difference between WT and TG livers. C: Quantification of PCNA-positive hepatocytes at 14 and 28 days of DDC feeding shows a significant increase in TG livers at 28 days compared with WT livers. ${ }^{*} P<0.01$. 
exhibited a ductular appearance, A6-positive cells resembling hepatocytes were increasingly evident in TG livers. To verify that these cells were indeed hepatocytes, we used double immunofluorescence and identified colocalization of $A 6$ and $C E B P \alpha$, a liver-enriched transcription factor and a hepatocyte marker (Figure 1B). Furthermore, the number of A6-positive hepatocytes (denoted "atypical hepatocytes" in the rest of this study) seemed to be greater in TG liver. Indeed, quantification of only atypical hepatocytes showed a significant increase at 14 days and, more notably, at 28 days in the TG liver (Figure 1D). Coincidentally, there is an increase in PCNA-positive hepatocytes, but not in biliary epithelial cells, in the TG mice compared with the WT mice at 28 days, suggesting that the atypical hepatocytes may exhibit a greater proliferative capacity (Figure 2, A-C). No differences in fibrosis were observed between the two groups over 3 to 28 days of DDC diet feeding as measured by trichrome staining (Figure 2A).

\section{Long-Term Exposure to DDC Leads to a High Number of Atypical Hepatocytes in TG Mice}

We next fed the diet for an extended period of time (150 days) to examine whether the appearance of atypical hepatocytes would continue and whether or not this response would have any effect on the chronic injury or repair process. After long-term exposure to DDC in WT mice, we observed significant periportal and pericellular fibrosis along with abnormal levels of bilirubin and alkaline phosphatase, indicating continued liver injury and increased intrahepatic cholestasis (Figure 3A, Table 1). Intriguingly, although WT livers do show the presence of atypical hepatocytes after 150 days of feeding of the DDC diet, a striking increase in their numbers was apparent in the TG livers (Figure 3A). On quantification, we observe nearly twice as many atypical hepatocytes in TG liver compared with WT liver (Figure 3B). In addition, although such hepatocytes were restricted to periportal areas in WT livers, their location was more widespread in the TG livers. Further analysis revealed several of such atypical hepatocytes to be exhibiting cytoplasmic and nuclear localization of $\beta$-catenin in the TG livers, a finding not observed in WT livers (Figure 3A).

Increased Atypical Hepatocytes in TG Livers Are Associated with a Decrease in the Markers of Intrahepatic Cholestasis after Long-Term DDC Feeding

To address any associated phenotypic association of enhanced atypical hepatocyte response to the chronic injury in the TG livers, we performed further histological analysis and serum biochemistry. Coincident with atypical hepatocytes, we observed greater hepatocyte proliferation in the
A

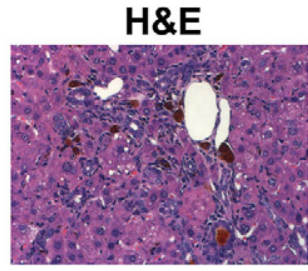

TG

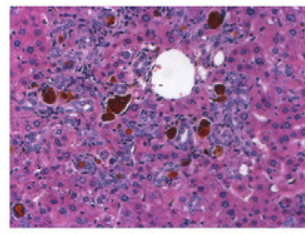

A6
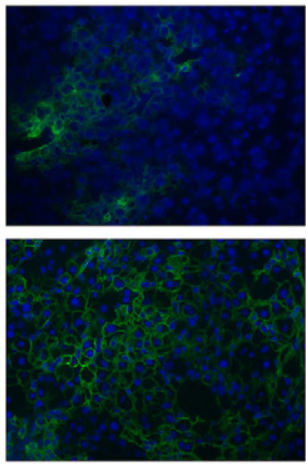

Beta-catenin
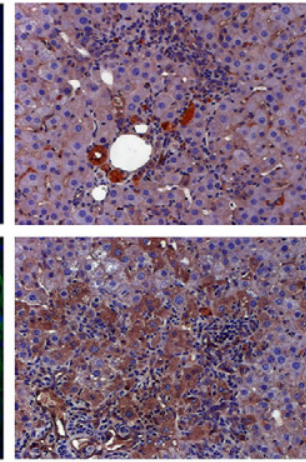

Trichrome

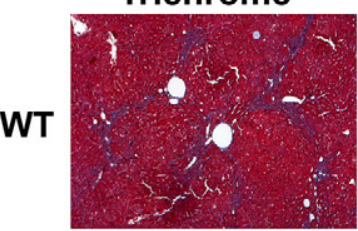

TG

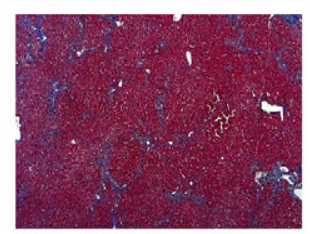

PCNA
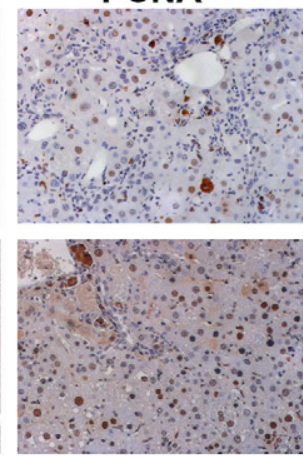

B

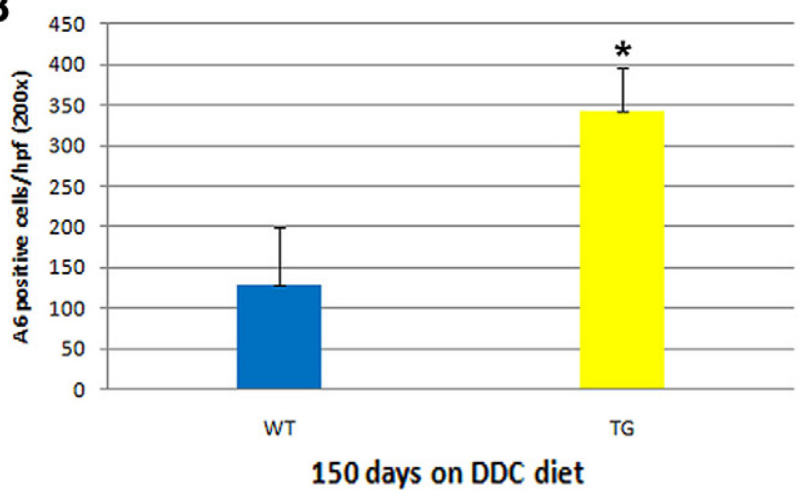

C

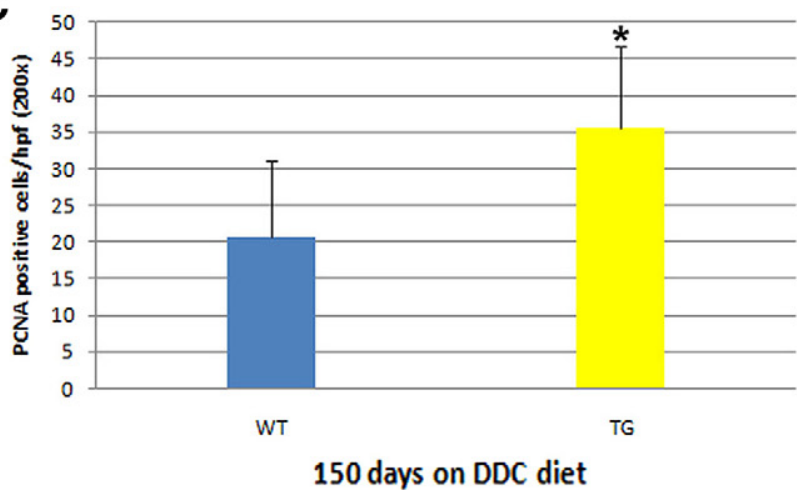

Figure 3. Continued increases in atypical hepatocytes and a decrease in the markers of biliary injury in TG livers after long-term DDC feeding. A: Representative photomicrographs for H\&E, A6, trichrome, and PCNA staining of livers from WT and TG mice after 150 days of DDC diet feeding. Original magnification: $\times 200$ (H\&E, A6, and PCNA); $\times 50$ (trichrome). 4',6-Diamidino-2-phenylindole (blue) was used to stain nuclei in immunofluorescence images. B: Quantification of A6-positive hepatocytes at 150 days of DDC feeding shows a significant increase in TG compared with WT livers. C: Quantification of PCNA-positive hepatocytes at 150 days of DDC feeding shows a significant increase in TG compared with WT livers. ${ }^{*} P<0.01$ 
Table 1. Serum Biochemistry after 150 Days of DDC Diet Feeding

\begin{tabular}{lcc}
\hline Serum biochemistry & WT & TG \\
\hline Total bilirubin & & \\
1 & 0.5 & 0.7 \\
2 & 2.9 & 0.2 \\
3 & 0.4 & 0.1 \\
4 & 5.1 & 0.8 \\
5 & 3.7 & 0.5 \\
Average & 2.52 & 0.46 \\
Alkaline phosphatase & & \\
1 & & 1397 \\
2 & 2323 & 1143 \\
3 & 2001 & 1113 \\
4 & 2199 & 1748 \\
5 & 3093 & 1935 \\
Average & 2404 & $1467.2^{*}$ \\
AST & \\
1 & & 2749 \\
2 & 2223 & 1332 \\
3 & 2134 & 1703 \\
4 & 1877 & 2649 \\
5 & 2256 & 2590 \\
Average & 2597 & 2204.6 \\
ALT & 2217.4 & \\
1 & & 3673 \\
2 & 2850 & 1219 \\
3 & 2544 & 1966 \\
4 & 2068 & 2914 \\
5 & 2675 & 2597 \\
Average & 3223 & 2473.8 \\
\hline
\end{tabular}

${ }^{*} P<0.05$.

TG livers as measured by PCNA staining, suggesting that these cells have an increased capacity for proliferation and regeneration (Figure $3, \mathrm{~A}$ and $\mathrm{C}$ ). Interestingly, serum analysis revealed that although bilirubin was abnormally elevated in three of five WT animals, all of the TG animals ( $n=$ 5) exhibited completely normal levels of total bilirubin (Table 1). Likewise, serum alkaline phosphatase levels are decreased by approximately $40 \%$ in the TG mice compared with those in the WT mice at 150 days (Table 1). In contrast to an improvement in the markers of intrahepatic cholestasis, no change in serum measurements of hepatocyte injury, AST, and ALT was observed (Table 1). We also examined the WT and TG livers at this stage for hepatic fibrosis through Masson trichrome staining, which showed unremarkable differences between the two groups (Figure 3A). Taken together, the above findings suggest that overexpression of $\beta$-catenin in hepatocytes, which leads to an enhanced atypical hepatocyte response to the long-term exposure to DDC, played a specific role in ameliorating intrahepatic cholestasis.

\section{TG Livers Resolve DDC-Induced Injury More Rapidly After Reinstitution of Normal Chow}

To further verify that excess $\beta$-catenin provided the liver with the capability to better repair injury, we performed DDC diet recovery studies. It was previously reported that the injury associated with DDC feeding resolves after the animal is fed normal chow again. ${ }^{19}$ We fed TG and WT mice the DDC diet for 28 days at which time normal chow was substituted for
Table 2. Serum Biochemistry for DDC-Induced Injury Recovery Study

\begin{tabular}{lcl}
\hline Serum biochemistry & WT & TG \\
\hline Total bilirubin & & \\
1 & 0.6 & 0.2 \\
2 & 0.4 & 0.2 \\
3 & 0.2 & 0.2 \\
4 & 0.3 & 0.1 \\
Average & 0.375 & 0.175 \\
Alkaline phosphatase & & \\
1 & 1269 & 928 \\
2 & 1122 & 902 \\
3 & 1108 & 996 \\
4 & 986 & 809 \\
Average & 1121.25 & $908.75^{*}$ \\
AST & & \\
1 & 737 & 596 \\
2 & 675 & 531 \\
3 & 875 & 697 \\
4 & 621 & 633 \\
Average & 727 & 614.25 \\
ALT & & \\
1 & 778 & 681 \\
2 & 622 & 666 \\
3 & 993 & 738 \\
4 & 726 & 831 \\
Average & 779.75 & 729 \\
\hline$*<0.05$. & & \\
& &
\end{tabular}

2 days. Such a switch led to normalization of serum bilirubin levels in both the WT and TG mice, although the values were consistently lower in the TG mice (Table 2). However, serum alkaline phosphatase levels were significantly lower in TG mice than in WT mice (Table 2). To determine whether the decline in intrahepatic cholestasis also led to histological improvement, we compared the extent of ductular proliferation and periportal inflammation in WT and TG livers after cessation of the DDC diet for 48 hours. Analogous to serum biochemistry, there seemed to be lower ADP in the TG livers than in the WT livers by H\&E staining (Figure 4A). Indeed, by immunofluorescence, there was a dramatic decrease in A6-positive atypical ductular cells in the TG livers (Figure 4, A and B). We also observed ongoing periportal inflammation in the WT livers, whereas periportal inflammation had mostly subsided in TG livers. This result was confirmed by CD45 staining, which showed many less infiltrating cells in the periportal zone of the TG livers (Figure 4, A and $\mathrm{C}$ ). Interestingly, TG and WT livers continued to show ongoing hepatocyte injury with serum AST and ALT levels being approximately 15 - to 18-fold higher than normal (Table 2). These findings reiterate the fact that overexpression of $\beta$-catenin in the hepatocytes provided a greater capacity to repair periportal injury associated with DDC exposure.

\section{Analysis of Differentiation Markers After Long-Term DDC}

To further address the role of cellular disparity in the form of increased numbers of atypical hepatocytes in the TG mice in promoting resolution of intrahepatic cholestasis, we began to examine various markers of differentiation in the TG and WT liver. Hepatocytes do not normally express the marker A6, which in the adult liver is only 
A
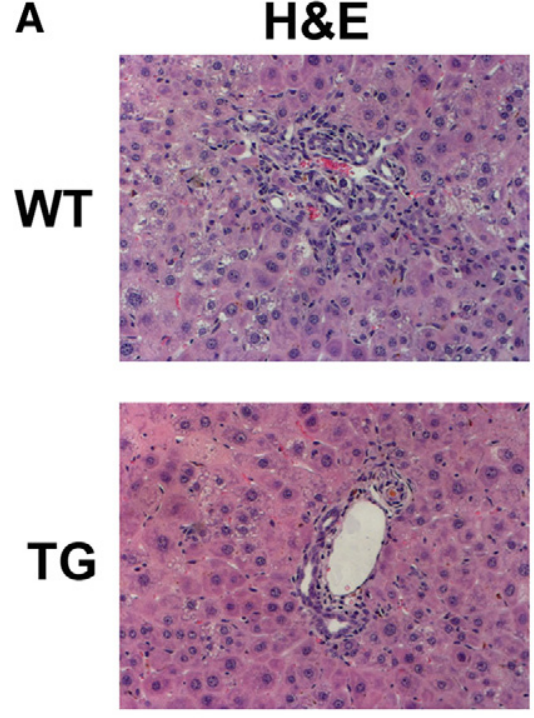

B

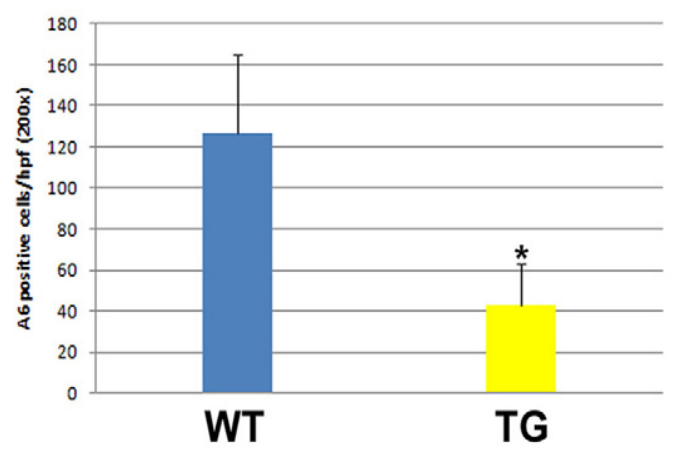

A6
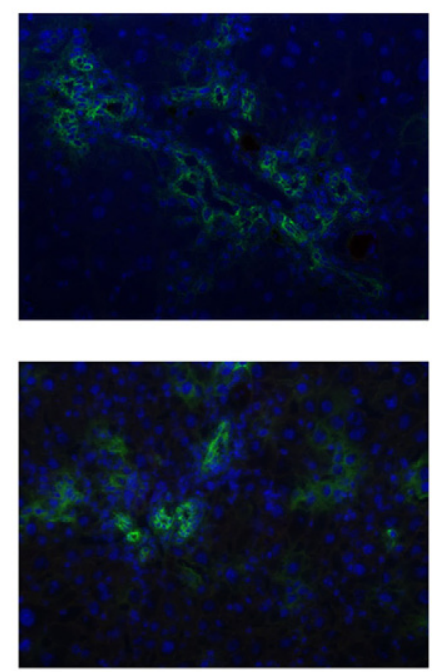

C

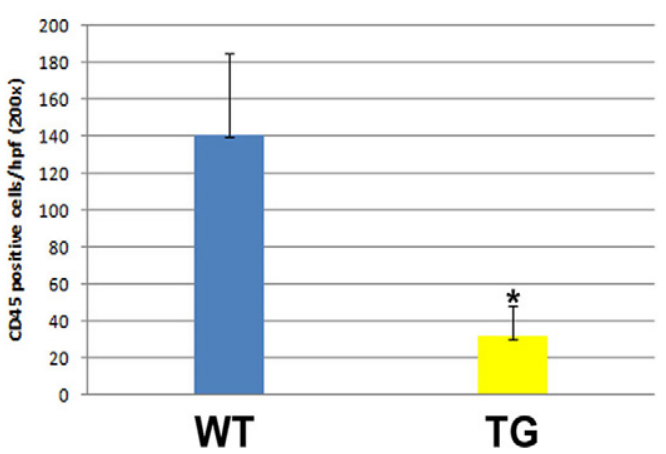

Figure 4. Overexpression of $\beta$-catenin enhances recovery from DDC diet-induced biliary injury. The DDC diet was fed to mice for 28 days and substituted with normal chow for 48 hours. A: Representative photomicrographs for H\&E, A6, and CD 45 staining of livers from WT and TG mice after 48 hours of recovery with a normal diet. Original magnification, $\times 2$. B: Quantification of A6-positive ductular cells shows a decrease in TG compared with WT liver. C: Quantification of CD45-positive cells shows a decrease in TG compared with WT liver. ${ }^{*} P<0.01$.

expressed in the biliary epithelial cells. However, the abundance of A6-positive hepatocytes in TG mice led us to believe that the differentiation status of these cells is altered. First, we examined expression of $\alpha$-fetoprotein (AFP), a marker found in fetal hepatocytes, after longterm DDC exposure. Both WT and TG livers show expression of AFP in hepatocytes, but expression is more widespread in TG livers (Figure 5A). Proliferating ductular cells do not express AFP. In contrast to AFP, Trop 2, a marker specific to the oval cell, was present in proliferating ductular epithelium with some periportal hepatocytes also retaining its expression in WT livers, whereas Trop-2 was predominantly localized only to the reactive ductular epithelium in TG (Figure 5A). ${ }^{20}$ CK19 was only expressed in ductular epithelial cells and not in hepatocytes in both WT and TG livers (Figure 5B). We also examined expression of $\mathrm{HNF} 1 \beta$, a transcription factor observed in both the bipotential hepatoblast as well as mature biliary epithelial cells. Expression of this maker was predominantly observed in the ductular epithelium in both groups (Figure 5B). However, some periportal hepatocytes retained nuclear HNF1 $\beta$ expression in WT livers only. Thus, we observe retention of some biliary and oval cell markers in WT livers whereas TG livers did not show such zonality, although they showed pronounced panzonal expression of $A 6$ and AFP.

\section{Discussion}

In the current study we examined the response to short- and long-term DDC diets in our mouse model expressing the $\beta$-catenin transgene in hepatocytes under the albumin promoter. The DDC diet model has been used in mice for various purposes including Mallory-Denk body formation and oval cell induction and was also reported as a model for inducing primary sclerosing cholangitis. ${ }^{15-19,21,22}$ After exposure to DDC, primarily biliary injury occurs, followed by hepatocyte injury. The injury is associated with overexpression of proinflammatory and profibrotic cytokines, elevation of markers of hepatic injury, periductal neutrophil infiltration, myofibroblast activation and proliferation, and subsequent periportal fibrosis. Eventually, chronic DDC exposure leads to dramatically larger livers, sometimes constituting up to $20 \%$ of body weight as has also been described elsewhere. ${ }^{16}$ In addition, there is continued abnormal serum biochemistry depicting chronic hepatic insult. In fact, DDC exposure for 150 days produced approximately 50-fold 
A

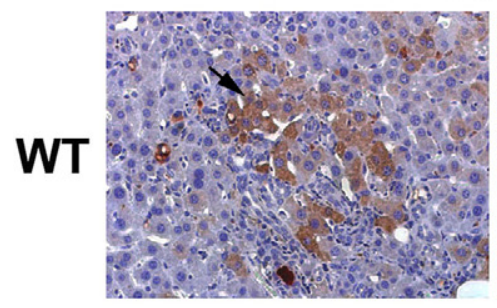

AFP
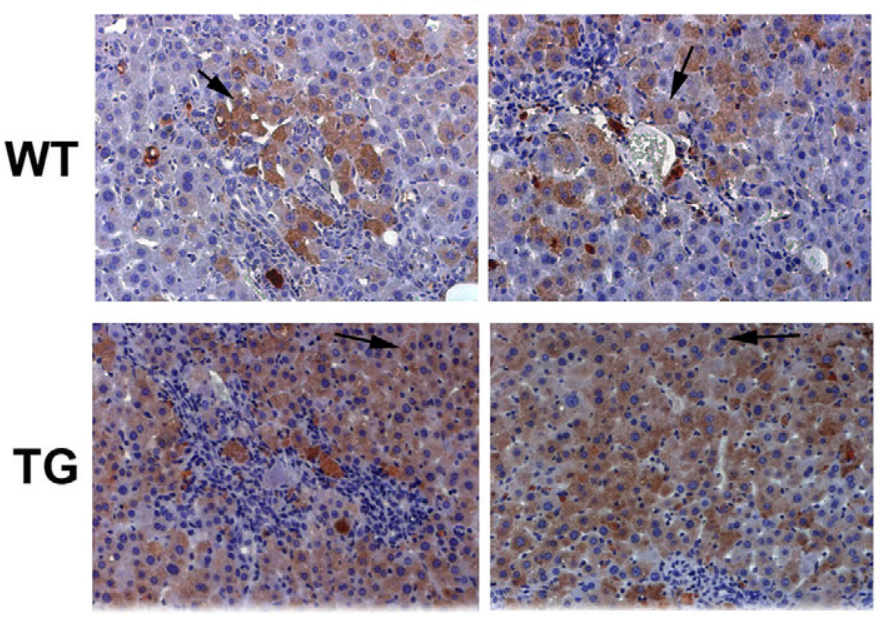

B

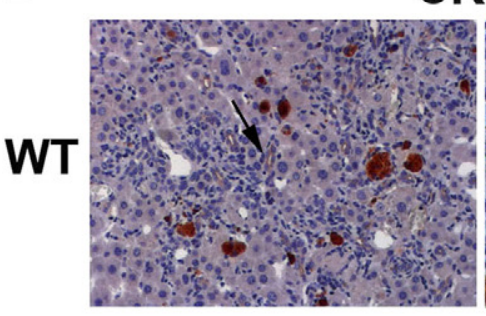

\section{CK19}
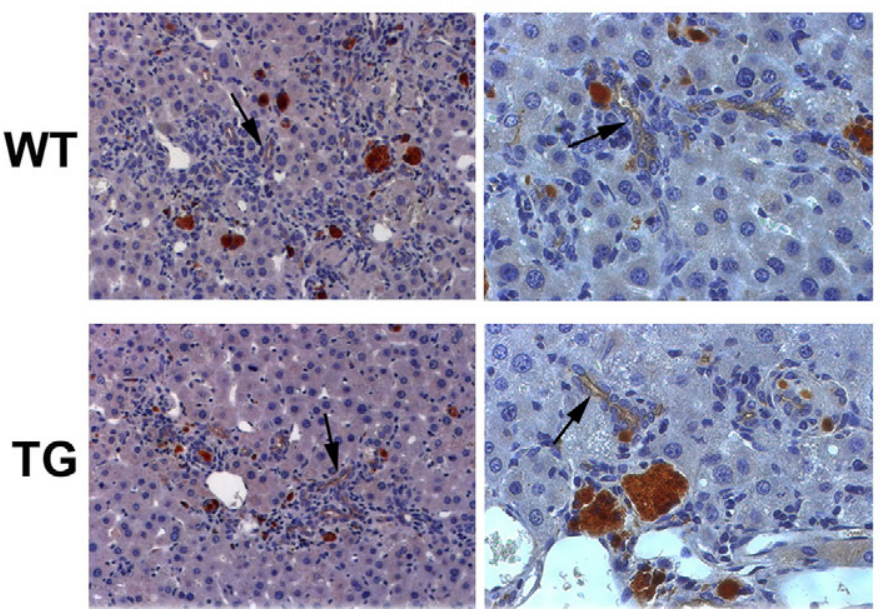

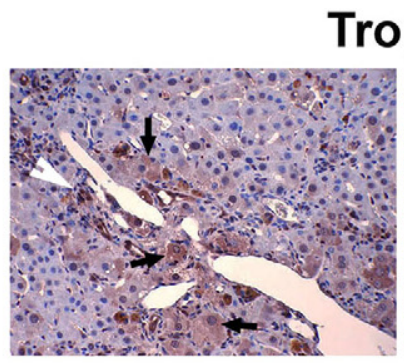

Trop-2
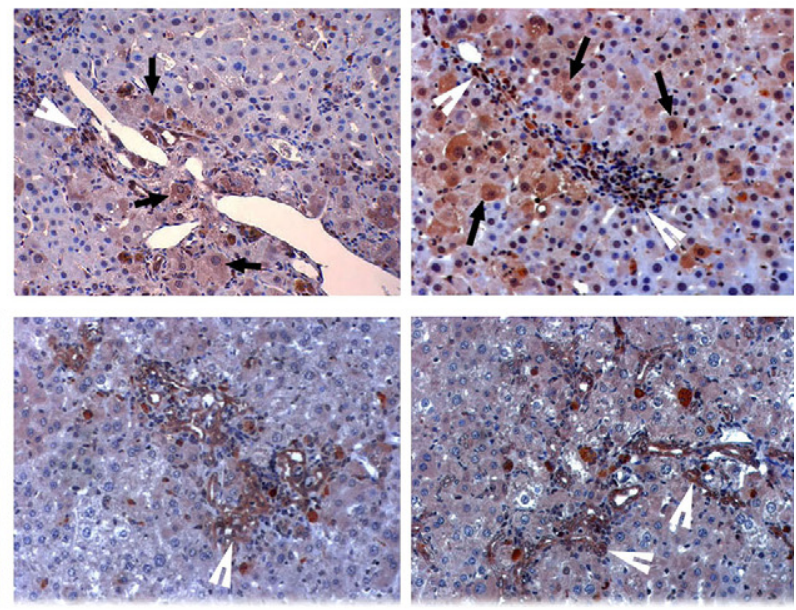

HNF1beta
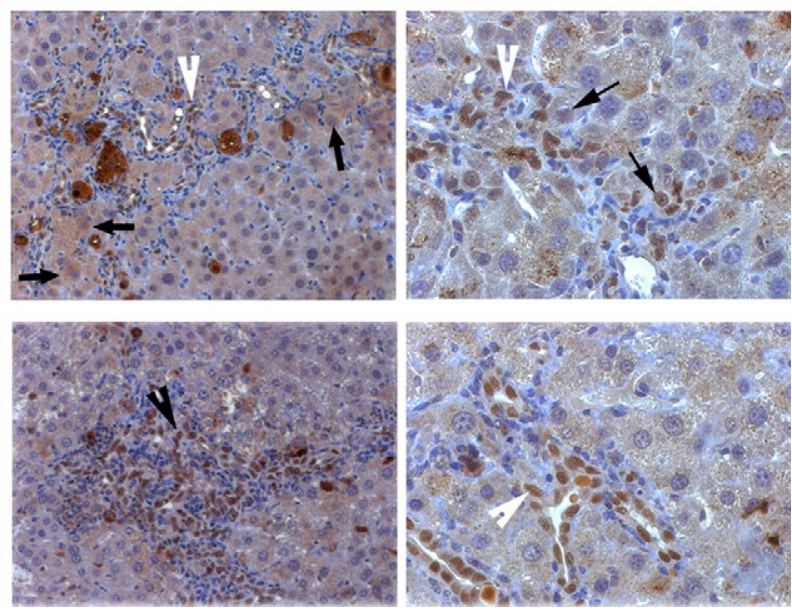

Figure 5. Expression analysis of markers of differentiation in liver after long-term DDC (150 days). A: Representative photomicrographs from two WT and two TG mice after long-term DDC exposure for AFP and Trop-2 staining. Whereas AFP-positive cells (arrows) are periportal in WT livers, they are panzonal in TG livers. WT livers show Trop-2-positive reactive ductules (white arrowhead) and periportal hepatocytes (arrows), whereas TG livers display Trop-2-positive ductules only (white arrowhead). Original magnification, $\times 200$. B: Representative photomicrographs for CK19 show only bile ducts to be positive (arrows) in WT and TG livers. HNF1 $\beta$ immunohistochemistry shows nuclear expression in atypical ductules (white arrowhead) and periportal hepatocytes (arrows) in WT livers but only in reactive ductules (white arrowhead) in TG livers after long-term DDC treatment. Original magnification, $\times 200$.

higher levels of serum AST and ALT levels in FVB mice. At the same time, these strains of mice showed significant intrahepatic cholestasis reflected by approximately 24-fold higher levels of alkaline phosphatase and approximately 2.5-fold excess levels of total bilirubin.

Despite massive injury, chronic DDC exposure is compatible with life for at least 5 to 6 months, which was the longest time point examined in our study. This seems to be due to diverse reparative responses in the form of ADP and hepatocyte proliferation. ${ }^{16,17}$ In addition, DDCinduced injury incites an oval cell response that emanates from ADP, and these progenitors differentiate into biliary epithelial cells and hepatocytes. ${ }^{16}$ Various specific or nonspecific markers for oval cells have been described, such as A6 and Trop-2, whereas others such as AFP and albumin are less well characterized. ${ }^{15-17,20,23}$

In the current study we sought to explore how $\beta$-catenin transgene expression in hepatocytes might influence the oval cell response and how it might affect the overall repair process after chronic exposure to DDC through examination of histology and serum biochemistry. The premise behind the study was a role of $\beta$-catenin in oval cells in rat and mouse models previously reported by others and us. ${ }^{13,14}$ In mice, $\beta$-catenin was shown to colocalize with the A6 population of cells, and there was induction of multiple Wnt ligands after DDC exposure. More definitively, a blunted ADP and oval cell response was reported in the conditional $\beta$-catenin-null liver. These findings led us to hypothesize that overexpression of $\beta$-catenin may induce a robust ADP and oval cell response after short-term exposure to DDC. Surprisingly, quantitative differences between the numbers of total A6-positive cells between the TG and WT livers were unremarkable from 3 to 28 days of DDC feeding. A possible explanation is that the $\beta$-catenin transgene is under the control of an albumin promoter/enhancer and oval cells may not express albumin, especially at the outset, which by itself is debatable. ${ }^{24,25}$ However, although there are no dramatic differences in ADP or oval cell numbers, we noted a mor- 
phological disparity between the A6-positive populations. The TG livers clearly exhibited a greater numbers of A6positive atypical hepatocytes. The periportal presence of A6-positive hepatocytes in the immediate proximity of oval cells has been shown previously after DDC injury and seems to represent the newly differentiated hepatocyte population from the oval cells, which are temporally retaining the A6 marker. ${ }^{16}$ At 28 days of DDC treatment, the numbers of atypical hepatocytes in TG livers, although located periportally, were significantly greater than those in WT livers, albeit at this stage no differences in serum biochemistry were apparent. When DDC feeding was continued for a total of 150 days, most of the resident hepatocytes in TG livers were expressing $A 6$, whereas in WT livers the atypical hepatocytes continued to be restricted to periportal areas only. Intriguingly, at this time we observed greater improvement in the two markers of intrahepatic cholestasis, serum bilirubin and alkaline phosphatase, in the TG liver, whereas hepatocyte injury and fibrosis seemed to be unaffected by overexpression of $\beta$-catenin. Thus, the $\beta$-catenin transgene in hepatocytes led to an enhancement of the atypical hepatocyte response coincident with improved resolution of intrahepatic cholestasis, mostly after long-term DDC exposure. This result also suggests that a minimal atypical hepatocyte response is essential for an improvement in hepatic function because serum biochemistry was unremarkable between WT and TG livers at 30 days after DDC exposure.

What is the mechanism of repair and how distinct is it in the WT and TG groups in response to long-term DDC? As mentioned above, DDC induces injury to bile ducts followed by hepatocytes. Although hepatocytes and biliary epithelial cells are facultative stem cells for themselves and for each other and the possibility of this phenomenon playing some role in the overall repair process after DDC injury cannot be ruled out, the chief mode of repair in this model seems to be ADP and oval cell response, the latter being the cell type differentiating into hepatocytes (Figure 6). This hypothesis is strengthened by the finding that only periportal hepatocytes in WT animals exposed to DDC are retaining some of markers of bile ducts (A6 and HNF1 $\beta$ ), oval cells (Trop-2), and immature hepatocytes (AFP) and as they mature, these hepatocytes lack such markers as evident in cells located away from the portal tract.

Although the foregoing process may be playing a role in hepatic repair in TG mice, there was a drastic difference in the cellular composition of TG livers after chronic DDC exposure. The $\beta$-catenin transgene in hepatocytes induced an overwhelming atypical hepatocyte response after DDC exposure. These atypical hepatocytes expressed nuclear and cytoplasmic $\beta$-catenin and some biliary (A6) and immature hepatocyte (AFP) markers and lacked expression of the specific oval cell marker Trop-2 and typical biliary markers CK19 and HNF1 $\beta$. More importantly, atypical hepatocytes are present panzonally after chronic DDC exposure and are not just localized to periportal areas. Thus, the mode of repair seems disparate in the TG livers and may represent any of the following scenarios above the WT response. The atypical hepatocyte response in TG livers may represent a more plastic

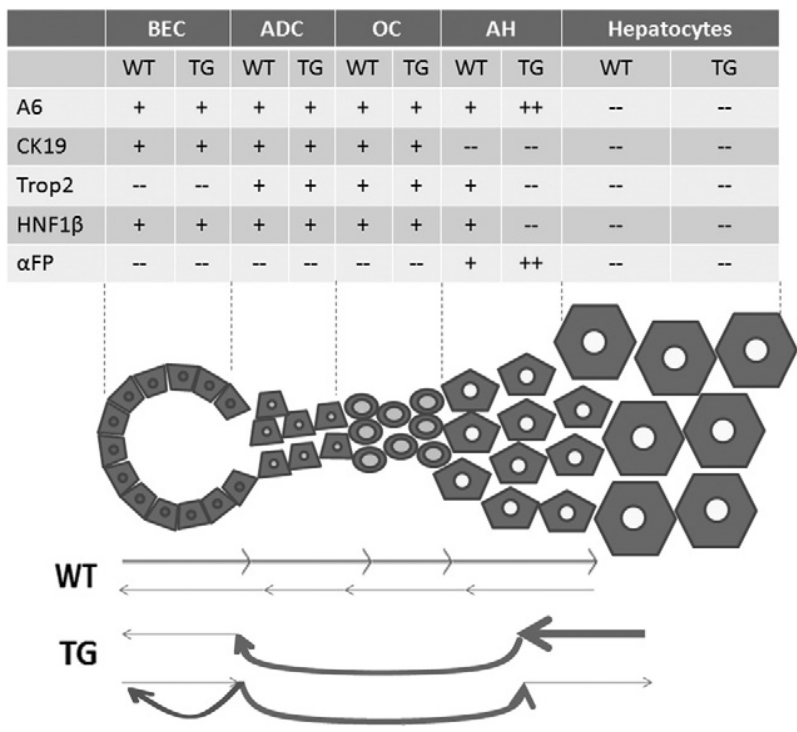

Figure 6. Cartoon depicting disparate modes of repair after long-term DDC-exposure in WT and TG livers. Although bidirectional repair from surviving bile ducts and hepatocytes ensures survival after chronic DDC injury that induces biliary and hepatocyte damage, the predominant mechanism seems to be through ductular proliferation, oval cell activation, and transdifferentiation to hepatocytes in WT livers. In TG livers, however, increased numbers of A6- and AFP-positive atypical hepatocytes panzonally and lack of periportal retention of biliary markers indicate enhanced transdifferentiation of $\beta$-catenin transgene-expressing hepatocytes to the biliary phenotype, eventually resolving intrahepatic cholestasis. An alternate scenario of increased differentiation to biliary cells and "spillover" to form A6-positive hepatocytes on expression of $\beta$-catenin transgene in oval cells may also contribute to the resolution of biliary injury.

hepatocyte that is able to resolve intrahepatic cholestasis and improve biliary function (lower serum bilirubin and alkaline phosphatase in TG livers) without affecting the extent of hepatic injury (comparable serum AST and ALT levels in WT and TG livers) (Figure 6). Indeed, it has been reported that hepatocytes are capable of transitioning into biliary epithelial cells without dedifferentiating through a bipotential oval cell or hepatic progenitor. ${ }^{26,27}$ Likewise culture of embryonic livers in serum-free conditions in the presence of Wnt3a-conditioned media, resulted in survival of biliary epithelial cells only, but more importantly resulted in transdifferentiation of remnant hepatocytes into biliary epithelium without their undergoing dedifferentiation as well. ${ }^{28}$ These studies fit well with the panzonal expression of A6 and AFP and predominant periportal atypical hepatocytes lacking biliary markers such as Trop-2 or HNF1 $\beta$, suggesting hepatocyte to biliary and not biliary to oval cell to hepatocyte directionality. Another possibility is that A6and Trop-2-positive oval cells on acquisition of albumin expression, which in turn induces expression of the $\beta$-catenin transgene, might favor an overall biliary phenotype, thus resolving cholestasis and improving biliary function. In this scenario, the A6-positive atypical hepatocytes essentially represent a "spillover" of the spare de novo-differentiated biliary cells that retain A6 but do not express HNF1 $\beta$ or CK19 and accumulate in the parenchyma over an extended period of chronic DDC ingestion and unremitting injury (Figure 6). Previous studies in hepatic development have shown an important role of $\beta$-catenin in regulating differentiation of hepatoblasts toward a biliary lineage. ${ }^{10,29}$ To con- 
clusively address the origin of A6-positive atypical hepatocytes in the TG studies ultimately requires lineage-tracing studies.

A major complication inherent to the DDC model of liver injury that makes a linear interpretation of the reparative process more complicated is the fact that unlike some of the other rodent models of oval cell activation, this model is confounded by ongoing hepatocyte proliferation, and hence bidirectional repair of hepatocyte and biliary injury repair cannot be ruled out normally or in the TG situation as these two processes might not be completely mutually exclusive. ${ }^{16}$ In fact, it is conceivable that several of the above scenarios might be functioning in concert to contribute to the hepatic repair. However, it is clear that the presence of the $\beta$-catenin transgene in hepatocytes repairs the hepatic injury predominantly through resolution of the intrahepatic cholestasis, which was also affirmed by the recovery studies as reflected by significant decreases in inflammation, ADP, and serum alkaline phosphatase levels in TG mice with 2 days of substitution of the DDC diet by normal chow.

The expression of biliary markers in hepatocytes has also been reported in various human diseases of the biliary tract. For instance, periseptal hepatocytes in patients with extrahepatic biliary atresia and $\alpha$-1-antitrypsin deficiency show expression of the maker OV-6, a finding also observed in hepatocytes from adult patients with primary biliary cirrhosis and sclerosing cholangitis. ${ }^{30,31}$ It was also reported that hepatocytes in the setting of cholestasis begin to show expression of the bile duct marker CK7. ${ }^{32}$ Interestingly, long-standing cholestasis led to an increase in the number of hepatocytes that were expressing CK7, much like what we observed with A6 expression in hepatocytes after long-term DDC. This result suggests that the appearance of hepatocytes expressing biliary makers increases with the amount of time the liver experiences biliary injury. Thus, chronic injury presents the need for more and more hepatocytes to be called into action in the repair and regenerative process.

If increased activity of the Wnt pathway provides the liver with an advantage for repairing the biliary epithelium, be it through increased hepatocytes plasticity or some other mechanism, this may be of clinical significance in the treatment of diseases that involve chronic cholestatic injury as a primary or secondary component. It is possible that therapy to induce Wnt pathway activation in these patients could have some benefit in the resolution of cholestasis. If this approach is eventually deemed feasible, studies must also include determination of the benefits of inducing the Wnt pathway for injury repair versus the cost of potentially promoting tumor development given the well studied oncogenic role of $\beta$-catenin in liver cancer. ${ }^{33}$

\section{References}

1. Roskams TA, Theise ND, Balabaud C, Bhagat G, Bhathal PS, BioulacSage P, Brunt EM, Crawford JM, Crosby HA, Desmet V, Finegold MJ, Geller SA, Gouw AS, Hytiroglou P, Knisely AS, Kojiro M, Lefkowitch JH, Nakanuma Y, Olynyk JK, Park YN, Portmann B, Saxena R, Scheuer PJ, Strain AJ, Thung SN, Wanless IR, West AB: Nomencla- ture of the finer branches of the biliary tree: canals, ductules, and ductular reactions in human livers. Hepatology 2004, 39:1739-1745

2. Libbrecht L, Desmet V, Van Damme B, Roskams T: Deep intralobular extension of human hepatic 'progenitor cells' correlates with parenchymal inflammation in chronic viral hepatitis: can 'progenitor cells' migrate? J Pathol 2000, 192:373-378

3. Lowes KN, Brennan BA, Yeoh GC, Olynyk JK: Oval cell numbers in human chronic liver diseases are directly related to disease severity. Am J Pathol 1999, 154:537-541

4. Imoto M, Nishimura D, Fukuda Y, Sugiyama K, Kumada T, Nakano S: Immunohistochemical detection of $\alpha$-fetoprotein, carcinoembryonic antigen, and ferritin in formalin-paraffin sections from hepatocellular carcinoma. Am J Gastroenterol 1985, 80:902-906

5. Van Eyken P, Sciot R, Paterson A, Callea F, Kew MC, Desmet VJ: Cytokeratin expression in hepatocellular carcinoma: an immunohistochemical study. Hum Pathol 1988, 19:562-568

6. Wu PC, Lai VC, Fang JW, Gerber MA, Lai CL, Lau JY: Hepatocellular carcinoma expressing both hepatocellular and biliary markers also expresses cytokeratin 14, a marker of bipotential progenitor cells. J Hepatol 1999, 31:965-966

7. Libbrecht L, Desmet V, Van Damme B, Roskams T: The immunohistochemical phenotype of dysplastic foci in human liver: correlation with putative progenitor cells. J Hepatol 2000, 33:76-84

8. Libbrecht L, De Vos R, Cassiman D, Desmet V, Aerts R, Roskams T: Hepatic progenitor cells in hepatocellular adenomas. Am J Surg Pathol 2001, 25:1388-1396

9. Micsenyi A, Tan X, Sneddon T, Luo JH, Michalopoulos GK, Monga SP: $\beta$-Catenin is temporally regulated during normal liver development. Gastroenterology 2004, 126:1134-1146

10. Monga SP, Monga HK, Tan X, Mule K, Pediaditakis P, Michalopoulos GK: $\beta$-Catenin antisense studies in embryonic liver cultures: role in proliferation, apoptosis, and lineage specification. Gastroenterology 2003, 124:202-216

11. Tan X, Yuan Y, Zeng G, Apte U, Thompson MD, Cieply B, Stolz DB, Michalopoulos GK, Kaestner KH, Monga SP: $\beta$-Catenin deletion in hepatoblasts disrupts hepatic morphogenesis and survival during mouse development. Hepatology 2008, 47:1667-1679

12. Monga SP, Micsenyi A, Germinaro M, Apte $U$, Bell A: $\beta$-Catenin regulation during Matrigel-induced rat hepatocyte differentiation. Cell Tissue Res 2006, 323:71-79

13. Apte U, Thompson MD, Cui S, Liu B, Cieply B, Monga SP: Wnt $/ \beta$ catenin signaling mediates oval cell response in rodents. Hepatology 2008, 47:288-295

14. Hu M, Kurobe M, Jeong YJ, Fuerer C, Ghole S, Nusse R, Sylvester KG: Wnt/ $\beta$-catenin signaling in murine hepatic transit amplifying progenitor cells. Gastroenterology 2007, 133:1579-1591

15. Petersen BE, Grossbard B, Hatch H, Pi L, Deng J, Scott EW: Mouse A6-positive hepatic oval cells also express several hematopoietic stem cell markers. Hepatology 2003, 37:632-640

16. Wang X, Foster M, Al-Dhalimy M, Lagasse E, Finegold M, Grompe M: The origin and liver repopulating capacity of murine oval cells. Proc Natl Acad Sci USA 2003, 100(Suppl 1):11881-11888

17. Preisegger KH, Factor VM, Fuchsbichler A, Stumptner C, Denk H, Thorgeirsson SS: Atypical ductular proliferation and its inhibition by transforming growth factor $\beta 1$ in the 3,5-diethoxycarbonyl-1,4-dihydrocollidine mouse model for chronic alcoholic liver disease. Lab Invest 1999, 79:103-109

18. Nejak-Bowen KN, Thompson MD, Singh S, Bowen WC, Dar MJ, Khillan J, Dai C, Monga SP: Accelerated liver regeneration and hepatocarcinogenesis in mice overexpressing serine- 45 mutant beta-catenin. Hepatology, May;51(5):1603-1613

19. Fickert P, Stoger U, Fuchsbichler A, Moustafa T, Marschall HU, Weiglein AH, Tsybrovskyy O, Jaeschke H, Zatloukal K, Denk H, Trauner M: A new xenobiotic-induced mouse model of sclerosing cholangitis and biliary fibrosis. Am J Pathol 2007, 171:525-536

20. Okabe M, Tsukahara Y, Tanaka M, Suzuki K, Saito S, Kamiya Y, Tsujimura T, Nakamura K, Miyajima A: Potential hepatic stem cells reside in EpCAM+ cells of normal and injured mouse liver. Development 2009, 136:1951-1960

21. Hanada S, Strnad P, Brunt EM, Omary MB: The genetic background modulates susceptibility to mouse liver Mallory-Denk body formation and liver injury. Hepatology 2008, 48:943-952

22. Preisegger $\mathrm{KH}$, Stumptner $\mathrm{C}$, Riegelnegg D, Brown PC, Silverman JA, Thorgeirsson SS, Denk H: Experimental Mallory body formation 
is accompanied by modulation of the expression of multidrugresistance genes and their products. Hepatology 1996, 24: 248-252

23. Bennoun M, Rissel M, Engelhardt N, Guillouzo A, Briand P, WeberBenarous A: Oval cell proliferation in early stages of hepatocarcinogenesis in simian virus 40 large T transgenic mice. Am J Pathol 1993, 143:1326-1336

24. Koenig S, Probst I, Becker H, Krause P: Zonal hierarchy of differentiation markers and nestin expression during oval cell mediated rat liver regeneration. Histochem Cell Biol 2006, 126:723-734

25. Qin $\mathrm{AL}$, Zhou XQ, Zhang W, Yu H, Xie Q: Characterization and enrichment of hepatic progenitor cells in adult rat liver. World $J$ Gastroenterol 2004, 10:1480-1486

26. Michalopoulos GK, Barua L, Bowen WC: Transdifferentiation of rat hepatocytes into biliary cells after bile duct ligation and toxic biliary injury. Hepatology 2005, 41:535-544

27. Michalopoulos GK, Bowen WC, Mule K, Lopez-Talavera JC, Mars W: Hepatocytes undergo phenotypic transformation to biliary epithelium in organoid cultures. Hepatology 2002, 36:278-283

28. Hussain SZ, Sneddon T, Tan X, Micsenyi A, Michalopoulos GK,
Monga SP: Wnt impacts growth and differentiation in ex vivo liver development. Exp Cell Res 2004, 292:157-169

29. Decaens T, Godard C, de Reynies A, Rickman DS, Tronche F, Couty JP, Perret C, Colnot S: Stabilization of $\beta$-catenin affects mouse embryonic liver growth and hepatoblast fate. Hepatology 2008, 47:247-258

30. Crosby HA, Hubscher S, Fabris L, Joplin R, Sell S, Kelly D, Strain AJ: Immunolocalization of putative human liver progenitor cells in livers from patients with end-stage primary biliary cirrhosis and sclerosing cholangitis using the monoclonal antibody OV-6. Am J Pathol 1998, 152:771-779

31. Crosby HA, Hubscher SG, Joplin RE, Kelly DA, Strain AJ: Immunolocalization of OV-6, a putative progenitor cell marker in human fetal and diseased pediatric liver. Hepatology 1998, 28:980-985

32. Van Eyken P, Sciot R, Desmet VJ: A cytokeratin immunohistochemical study of cholestatic liver disease: evidence that hepatocytes can express 'bile duct-type' cytokeratins. Histopathology 1989, 15:125-135

33. Monga SP: Role of Wnt/ $\beta$-catenin signaling in liver metabolism and cancer. Int J Biochem Cell Biol 2009, DOI: 10.1016/ j.biocel.2009.09.001. 